\title{
Bovine Serum Albumin-Dependent Charge-Transfer Kinetics Controls the Electrochemical Immunosensitive Detection: Vibrio cholerae as a Model Bioanalyte
}

\author{
Okoroike C. Ozoemena ${ }^{1} \cdot$ Tobechukwu J. Ehirim $^{2} \cdot$ Tobile Khawula $^{2} \cdot$ Katlego Makgopa $^{3} \cdot$ Leshweni J. Shai $^{1}$. \\ Kenneth I. Ozoemena² ${ }^{2}$
}

Accepted: 26 May 2021 / Published online: 9 June 2021

(c) The Author(s), under exclusive licence to Springer Science+Business Media, LLC, part of Springer Nature 2021

\begin{abstract}
This work investigates how bovine serum albumin (BSA), a commonly used protein in the fabrication of electrochemical immunosensors, can impact on the sensitivity of detection when integrated with antibody $(\mathrm{Ab})$ pre-encapsulated with (i) insulating polyacrylonitrile (PAN) fibre (i.e., GCE-PAN-Ab-BSA immunosensor) or (ii) conducting PAN-grafted iron (II) phthalocyanine (FePc) (i.e., GCE-PAN@FePc-Ab-BSA immunosensor), using Vibrio cholerae toxin as a case study bioanalyte. Both immunosensors show different charge-transfer kinetics that strongly impact on their immunosensitive detection. From the electrochemical data, GCE-PAN-Ab-BSA is more insulating with the presence of BSA, while the GCE-PAN@ FePc-Ab-BSA is more conducting with BSA. The CV of the GCE-PAN-Ab-BSA is dominated by radial diffusion process, while that of the GCE-PAN@FePc-Ab-BSA is planar diffusion process. The behaviour of GCE-PAN@FePc-Ab-BSA has been associated with the facile coordination of BSA and FePc that permits co-operative charge-transport of the redox probe, while that of the GCE-PAN-Ab-BSA is related to the interaction-induced PAN-BSA insulating state that suppresses chargetransport. As a consequence of these different interaction processes, GCE-PAN-Ab-BSA immunosensor provides higher electroanalytical performance for the detection of Vibrio cholerae toxin (with sensitivity of $16.12 \Omega / \log [\mathrm{VCT}, \mathrm{g} / \mathrm{mL}$ ] and limit of detection (LoD) of $3.20 \times 10^{-13} \mathrm{~g} / \mathrm{mL}$ compared to those of the GCE-PAN@FePc-Ab-BSA (4.16 $\Omega / \log$ (VCT, g $\mathrm{mL}^{-1}$ ) and $2.00 \times 10^{-12} \mathrm{~g} / \mathrm{mL}$ ). The study confirms the need for a thorough understanding of the physico-chemistries of the electrode platforms for the construction of immunosensors. Although this work is on immunosensors for cholera infection, it may well apply to other immunosensors.
\end{abstract}

Keywords Vibrio cholerae detection · Bovine serum albumin (BSA) - Iron (II) phthalocyanine (FePc) · Polyacrylonitrile (PAN) fibre $\cdot$ Charge-transfer kinetics $\cdot$ Sensitivity

Okoroike C. Ozoemena

208141392@tut4life.ac.za

$\triangle$ Leshweni J. Shai

ShaiLJ@tut.ac.za

$\triangle$ Kenneth I. Ozoemena

Kenneth.ozoemena@wits.ac.za

1 Department of Biomedical Sciences, Faculty of Science, Tshwane University of Technology, Pretoria 0001, South Africa

2 Molecular Sciences Institute, School of Chemistry, University of the Witwatersrand, Johannesburg 2050, South Africa

3 Department of Chemistry, Faculty of Science, Tshwane University of Technology, Pretoria 0001, South Africa

\section{Introduction}

Cholera is a disease that demonstrates the global socio-economic inequalities. It is mostly the disease more prevalent in povertystricken communities. It is a bacterial disease that is characterized by diarrhoea, dehydration, vomiting, and death, especially if it is not quickly detected and treated. The causative agent of cholera is called Vibrio cholerae, a bacterium that produces cholera toxin. The onset of the infection with $V$. cholerae follows the ingestion of contaminated drinking water or food by humans. It was shown in a study conducted between 2000 and 2008 that about 1.4 billion of people were at the risk of cholera infection, and children over the age of 5 years were found to show symptoms of cholera infection [1]. The reported cholera infection across the globe remains between 1.3 and 4.0 million [2]. 
Several techniques exist for the diagnosis of cholera infection, with the culture method as the gold standard [3]. Unfortunately, culture method is still associated with several disadvantages, including huge time-consumption, high cost, and the requirement of well-trained specialists to carry out the analysis. For example, culture method can take up to 8 days to get the results while, according to literature [4], cholera kills within 12 to $24 \mathrm{~h}$ if not treated. There are many other methods such as enzyme-linked immunosorbent assay [5], polymerase chain reaction (PCR) [6, 7], and electrochemical methods [8-15]. Of all the aforementioned techniques, electrochemistry gives excellent promise for fast detection, simplicity, sensitivity, selectivity, and ability to miniaturize the process for the development of hand-held point-of-care diagnostic devices. There is a need to continue to research on the development of electrochemical methods for improved detection of cholera infection.

Generally, electrochemical immunosensors involve the modification of an electrode surface or platform with a specific antibody (or antigen) to allow for the interaction of the corresponding antigen (or antibody). The main attraction of immunosensors is their high specificity of interaction as only specific antibody interacts with its specific antigen in a lock-and-key manner. The electrode platform used in the fabrication of the immunosensors is a key determining factor for the sensitivity of detection. Thus, several researchers have reported several electrode platforms for the immobilization of cholera immunosensors. These electrode platforms include graphene [8], liposomes and poly (3,4ethylenedioxythiophene)-coated carbon nanotubes [9], dendrimers integrated with gold nanoparticles [10], zinc oxide [11], polyacrylonitrile (PAN) nanofibres [12], copper (II) complex with polypyrrole-nitrilotriacetic acid on carbon nanotubes [13], carbon nanofibres (CNF) [14], and onionlike carbon modified with PAN (OLC-PAN) [15]. In electrochemistry, to be redox-active, a material must be able to lose or gain electrons (i.e., conducting), while a redox-inactive (or redox-silent) material is insulating. Unlike inorganic materials, most polymeric materials are bio-compatible with antibodies and antigens; hence, their use as electrode platforms for immunosensors. Most studies on immunosensors, in particular those using polymeric electrode platforms (redox-active or redox-inactive), focus on determining the basic analytical parameters (such as detection limits and linear concentration range) with no interest or effort on understanding the scientific reasons for the differences in the analytical data. This is the key motivation for this work.

Bovine serum albumin (BSA) is an important protein which is popularly used to block non-specific sites in the immunosensing platforms [9-17]. In electrochemical immunosensors, the sensing mechanism is generally dominated by capacitance (i.e., non-Faradaic detection) due to the redox-inactivity of the electrode platform upon which the antibody or antigen is immobilized. Generally, the introduction of the BSA onto the electrode platforms (redox-active or redox-silent) enhances the insulating behaviour of the immuosensors. The question is "why is this so"? The answer is that BSA is unable to interact with the species that make up the electrode platform. So, the next question would be "What if the electrode platform contains a specie that can interact with BSA, leading to electrocatalysis or enhanced charge-transfer kinetics, to what extent can this affect the immunosensing detection"? To our knowledge, there is no study that has attempted to investigate nor answered this very important question. The answer to the question will allow researchers to make rational decision on the type of electrode platform that should be employed in constructing an immunosensor, in terms of the ability of the electrode material to enhance electrocatalysis (i.e., Faradaic response, or enhanced charge-transfer kinetics) or reduce it (i.e., insulating, or poor electron transfer kinetics).

In this work, for the first time, we investigate the use of polyacrylonitrile (PAN) fibre (which is non-conducting) and iron(II)phthalocyanine-polyacrylonitrile (PAN@FePc) fibre (which is conducting due to the presence of the FePc species) as electrode platforms for the immobilization of cholera antibody for the detection of Vibrio cholerae toxin (antigen). The work reveals, amongst other findings, that (i) both PAN and PAN@FePc exhibit different physico-chemical properties (in terms of morphology, surface areas, and pore volumes), which have direct impact on their voltammetry and limits of detection of Vibrio cholerae toxin, and (ii) that BSA interacts with redox-active FePc in PAN, leading to enhanced chargetransfer processes that are inimical to the sensitive detection of Vibrio cholerae toxin.

\section{Experimental}

\section{Materials and Methods}

All the reagents used were obtained from Sigma-Aldrich South Africa, and include the following: polyacrylonitrile, iron(II)phthalocyanine (FePc), glycine, 1-ethyl-3-(3dimethylaminopropyl) carbodiimide hydrochloride (EDC), N-hydroxysulfosuccinimide (Sulfo-NHS), cholera toxin B subunit antigen (Ag) (from Vibrio cholerae Cat. No. C9903, 95\% SDS-PAGE, lyophilized powder), anti-cholera toxin antibody $(\mathrm{Ab})$ (from rabbit, whole antiserum, Cat. No. C3062), bovine serum albumin (heat shock fraction, $\mathrm{pH}$ $7, \geq 98 \%$ purity), and thiosulphate citrate bile salt (TCBS). All the reagents for the experiments were prepared using ultrapure water (18.2 $\mathrm{M} \Omega \mathrm{cm}$ resistivity). Phosphate buffer, containing small amounts of sodium azide (as a preservative) and EDTA (for disentangling cells) (PBS/AE, pH 7.4), was prepared as described in the literature [18]. The redox 
probe $\left(0.1 \mathrm{M} \mathrm{K}_{4} \mathrm{Fe}(\mathrm{CN})_{6} / \mathrm{K}_{3} \mathrm{Fe}(\mathrm{CN})_{6}(1: 1\right.$ mixture $)$ was prepared using the PBS/AE, $\mathrm{pH}$ 7.4.

Electrospun polyacrylonitrile (PAN) fibre and iron (1l) phthalocyanine (FePc)/polyacrylonitrile fibre (PAN@FePc) hybrid were obtained by electrospinning procedure $[19,20]$. The procedure is summarized in Fig. 1.

First, the precursor materials (PAN or a mixture of PAN and FePc, 1:1 mixture) were dissolved in DMF at the ratio of $1 \mathrm{mg}$ PAN or PAN@FePc to $2 \mathrm{ml}$ DMF and properly ultrasonicated to form a solution. The solution was then injected through the syringe into the electrospinning machine at $10 \mathrm{kV}$ power supply and at room temperature at a flow rate of $0.6 \mathrm{~mL} / \mathrm{h}$. A distance of about $15 \mathrm{~cm}$ was maintained between the aluminum collector plate and the syringe point. The collected products (PAN fibre and PAN@FePc fibres) were collected, soaked in distilled de-ionized water for about $12 \mathrm{~h}$ to wash off the DMF, then oven-dried $\left(60^{\circ} \mathrm{C}\right.$ for $\left.2 \mathrm{~h}\right)$ to give the final products.

\section{Construction of the PAN and PAN@FePc-Based Electrochemical Immunosensors}

During the fabrication of the immunosensor, the conventional method of covalent linking of the antibody on the electrode platform was strictly adhered to as previously reported $[14,15]$. This is summarized as follows: the bare electrode (GCE) was cleaned, then the nanomaterial (e.g., PAN) dissolved in 10- $\mu \mathrm{L}$ DMF solution and drop dried on the bare GCE, which was slowly dried at $40{ }^{\circ} \mathrm{C}$ to form GCE-PAN. The GCE-PAN was immersed in a solution of $2 \mathrm{M} \mathrm{NaOH}$ for $2 \mathrm{~h}$ at $40{ }^{\circ} \mathrm{C}$, to expose the $-\mathrm{COOH}$ group for subsequent covalent bonding with EDC/sulfo-NHS mixture. Then, the carboxylated carbonaceous fragments were washed off on the electrode surface to enhance the

(a)

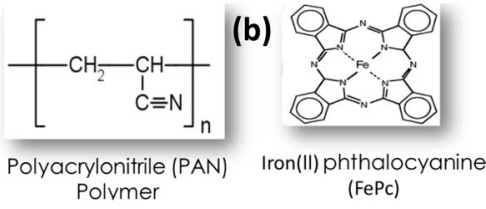

(c)

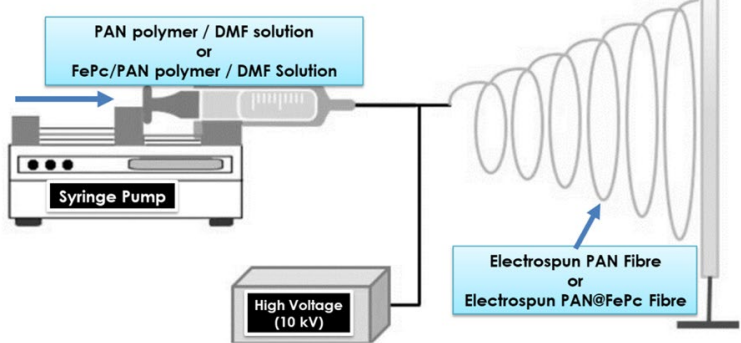

Fig. 1 Schematic representation of the electrospinning process for the preparation of PAN fibre and PAN@FePc fibre coordination of the $-\mathrm{NH}_{2}$ group of the Vibrio cholerae antibody ( $5 \mu \mathrm{L}$ from $1-\mathrm{mL}$ commercial solution) to form GCE-PAN-Ab. BSA was introduced to block non-specific sites of the porous electrode, resulting the final modified electrode abbreviated as GCE-PAN-Ab-BSA. The same process was also followed in the modification of PAN@ $\mathrm{FePc}$, and the final immunosensor produced was abbreviated as GCE-PAN@FePc-Ab-BSA. The final modified immunosensor was stored at $4{ }^{\circ} \mathrm{C}$ until use. The procedure for electrochemical immunosensor fabrication is summarized in Fig. 2 below.

\section{Electrochemical Procedures}

An Autolab Potentiostat PGSTAT 100 (Eco Chemie, Utrecht, The Netherlands) driven the General-Purpose Electrochemical System (GPES ${ }^{\circledR}$ version 4.9) was used for all the electrochemical experiments, including the cyclic voltammetry (CV) and electrochemical impedance spectroscopy (EIS). Glassy carbon electrode (GCE, diameter $=3.0 \mathrm{~mm}$, BAS $\left({ }^{\circ}\right)$ with or without the modifying materials, PAN and PAN@FePc, served as the working electrode, Pt rod, and $\mathrm{Ag} / \mathrm{AgCl}(3 \mathrm{M} \mathrm{KCl})$ served as the counter and reference electrodes, respectively. Before using the electrolyte for any experiment, it was first de-aerated with pure nitrogen (Afrox, South Africa), which is conducted at room temperature. Every EIS experiment was carried out with the GPES ${ }^{\circledR}$ Frequency Response Analyzer (FRA) software between $100 \mathrm{kHz}$ and $0.01 \mathrm{~Hz}$ with the amplitude (rms value) of the ac signal of $10 \mathrm{mV}$, in the electrolyte solution containing the redox probe $\left(0.1 \mathrm{M} \mathrm{K}_{4} \mathrm{Fe}(\mathrm{CN})_{6} / \mathrm{K}_{3} \mathrm{Fe}(\mathrm{CN})_{6}(1: 1 \mathrm{~mol}\right.$ ratio $)$ dissolved in $0.14 \mathrm{M} \mathrm{PBS} / \mathrm{AE}$ ( $\mathrm{pH}$ 7.4)). The EIS was performed at the equilibrium potential $\left(E_{1 / 2}\right)$ of the redox probe (ca. $0.13 \mathrm{~V}$ vs $\mathrm{Ag} \mid \mathrm{AgCl}, 3 \mathrm{M} \mathrm{KCl}$ ).

\section{Results and Discussion}

\section{Morphological Characterization}

Figure 3 compares the SEM images of PAN fibres (A) and PAN@FePc fibres (B). Both materials exhibit one-dimensional morphology, with the PAN fibres showing fine and smooth structure of about $\leq 0.5 \mu \mathrm{m}$ in diameter and several microns in length. The PAN@FePc fibres, on the other hand, gave rough fibres with an average diameter of $\geq 2.5 \mu \mathrm{m}$, which is more than five times greater than that of the average PAN fibre. Brunauer-Emmett-Teller (BET) analysis was conducted to further establish the physical properties of the PAN and PAN@FePc fibres. From the BET data, the 


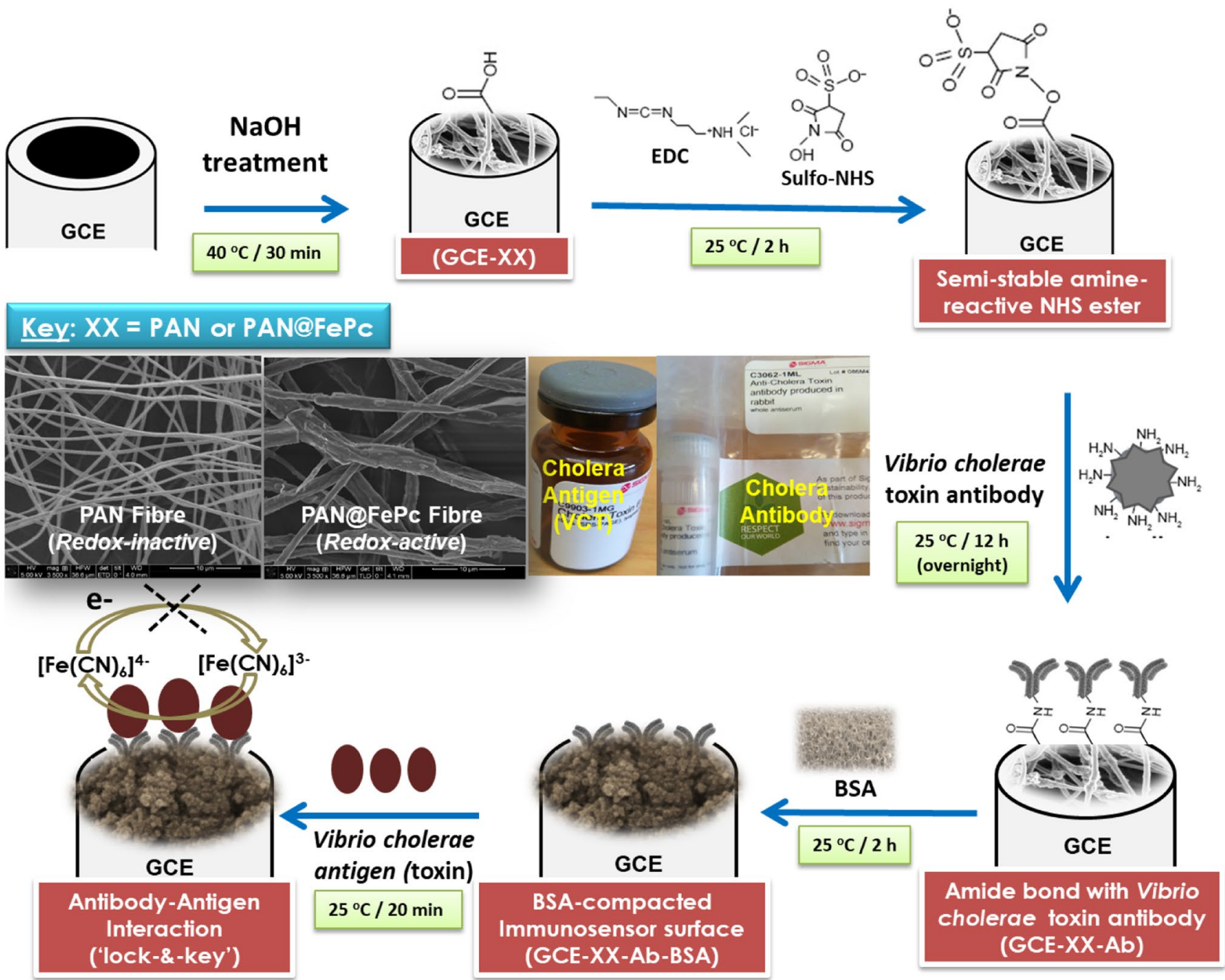

Fig. 2 The construction partway of the PAN fibre and PAN@FePc hybrid based electrochemical immunosensor for the detection of Vibrio cholerae toxin / antigen (VCT). Inset is a typical SEM image

FeP-PAN fibres exhibit surface area $\left(37.56 \mathrm{~cm}^{2} \mathrm{~g}^{-1}\right)$ and pore volume $\left(0.20 \mathrm{~cm}^{3} \mathrm{~g}^{-1}\right)$ which are more than twice those of the PAN fibres (i.e., $17.87 \mathrm{~cm}^{2} \mathrm{~g}^{-1}$ and $0.08 \mathrm{~cm}^{3} \mathrm{~g}^{-1}$ ).

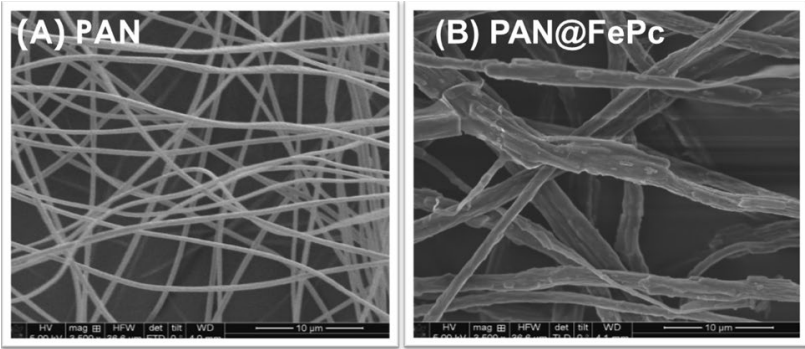

Fig. 3 Typical SEM images of the (A) PAN fibres, and (B) PAN@ FePc fibres of the PAN fibre / PAN@FePc used as the base electrode platform as well as the commercial Vibrio cholerae antibody and antigen

\section{Mass Transport and Electron Transfer Kinetics of the Two Different Immunosensors}

The Faradaic current that flows at an electrode surface is interpreted on two inexorably intertwined processes: (i) the mass transport (i.e., the diffusion rate of the analyte from the bulk electrolyte to the electrode) and (ii) charge-transfer kinetics (i.e., electronic or ionic transport across the electrode-electrolyte interface) [21]. Figure 4 compares the cyclic voltammograms (CVs; Fig. 4A, B) of the PAN and PAN@FePc before and after the introduction of BSA to obtain the final immunosensors. Table 1 summarizes the $\mathrm{CV}$ parameters of the electrodes, with and without the BSA.

The ratio of the anodic-to-cathodic peak current $\left(I_{\mathrm{pa}} / I_{\mathrm{pc}}\right)$ provides some insight into the kinetics of the electrodes; the best reversibility gives the ratio as unity. With the exception 


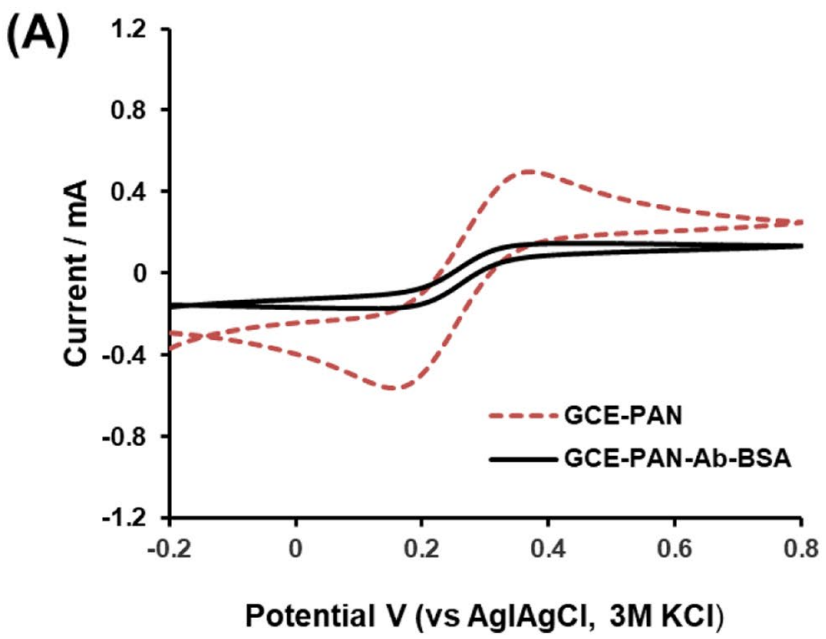

Fig. 4 Comparing the electrocatalytic data of the PAN and PAN@ FePc electrodes before and after the introduction of the BSA: Cyclic voltammograms of the electrode platforms obtained from the (A)

of the GCE-PAN-Ab-BSA, all the other electrodes showed excellent reversibility $\left(I_{\mathrm{pa}} / I_{\mathrm{pc}} \approx 1\right)$. The poor reversibility of the GCE-PAN-Ab-BSA $\left(I_{\mathrm{pa}} / I_{\mathrm{pc}} \approx 0.7\right)$ is indicative of restricted electron transport. Similarly, the electron-transfer kinetics are evident from the values of the peak-to-peak separation potential $\left(\Delta E_{\mathrm{p}} / \mathrm{V}\right)$; the smaller the value of the $\Delta E_{\mathrm{p}}$, the faster the rate of the electron-transfer. From the $\mathrm{CV}$ data of the constructed immunosensor containing the BSA, the FePc-based immunosensor (i.e., GCE-PAN@ FePc-Ab-BSA) exhibits faster electron-transfer kinetics $\left(\Delta E_{\mathrm{p}} \approx 0.20 \mathrm{~V}\right)$ than the immunosensor without the FePc (i.e., GCE-PAN-Ab-BSA: $\Delta E_{\mathrm{p}} \approx 0.22 \mathrm{~V}$ ). The increase in current response (mass transport) and electrode kinetics following the introduction of the antibody (observed in both redox-active and redox-inactive electrode platforms) is somewhat surprising considering that antibody is known to be bulky and not as conducting. However, similar observations have been made by Solanki and co-workers [11, 12] who attributed the behaviour to either the presence of the redox mediator in the electrode platform [12] or the porous nature of the electrode surface [11]. We believe that similar processes are taking place in this work but, in addition, mass

Table 1 Summary of the cyclic voltammetric data for the PAN and PAN@FePc hybrid with and without BSA (as shown in Fig. 4)

\begin{tabular}{llllll}
\hline Electrode & \multicolumn{5}{l}{ CV data } \\
\cline { 2 - 6 } & $I_{\mathrm{pa}} / \mu \mathrm{A}$ & $I_{\mathrm{pc}} / \mu \mathrm{A}$ & $I_{\mathrm{pa}} / I_{\mathrm{pc}}$ & $E_{1 / 2} / \mathrm{V}$ & $\Delta E_{\mathrm{p}} / \mathrm{V}$ \\
\hline GCE-PAN & 537 & -538 & 1.00 & 0.13 & 0.188 \\
GCE-PAN-Ab-BSA & 10.33 & -14.93 & 0.70 & 0.13 & 0.342 \\
GCE-PAN@FePc & 669.6 & -635.6 & 1.05 & 0.13 & 0.218 \\
GCE-PAN@FePc-Ab- & 866.1 & -841.7 & 1.03 & 0.13 & 0.196 \\
BSA & & & & & \\
\hline
\end{tabular}

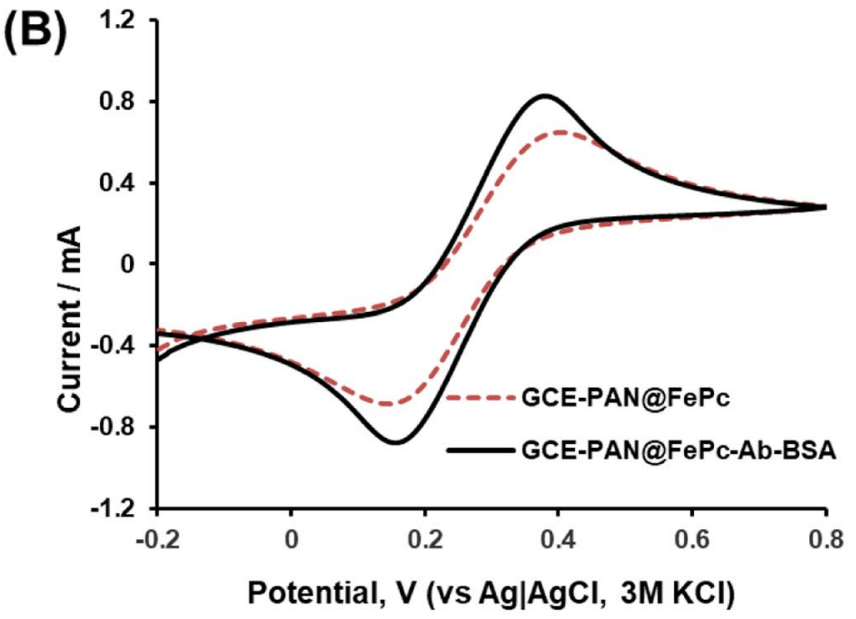

PAN-based sensors, and (B) PAN@FePc-based sensors. All data were collected in redox probe $\left(\left[\mathrm{Fe}(\mathrm{CN})_{6}\right]^{4-} /\left[\mathrm{Fe}(\mathrm{CN})_{6}\right]^{3-}\right)$ in PBS/AE (pH 7.4) at $50 \mathrm{mVs}^{-1}$

transport/diffusion modes (as described before [21]) and will be discussed below, are playing key electrochemical roles.

The CV data show that the presence of BSA strongly suppressed the current response at the GCE-PAN-Ab-BSA but enhanced that of the GCE-PAN@FePc-Ab-BSA. This result suggests that BSA easily interacts or co-operate with FePc to catalyze the redox activity of the probe, thus fast chargetransfer kinetics. On the contrary, with the PAN alone, the interaction with the BSA leads to a more insulating state that strongly suppresses charge-transfer kinetics. Surprisingly, this finding has rarely been reported in any BSA-incorporated electrochemical immunosensors. For example, Gupta et al. [11] observed higher electron transfer rate constants (highrate constant) with ITO-ZnO-Ab-BSA immunosensor than the corresponding electrodes without BSA (i.e., ITO-ZnO or ITO-ZnO-Ab) and attributed the finding only to the porous nature of the electrode surface. Similar to our case, PAN@ FePc is more porous than the PAN (see BET data in Table 1), but we believe that the redox kinetics of BSA in the presence of FePc is beyond the porous nature of the PAN@FePc electrode. From literature reports, the following are apparent: First, the adsorption of BSA with non-metal and polymeric materials is generally accompanied with increased chargetransfer resistance $\left(R_{\mathrm{CT}}\right)$ [22, 23]; thus, the poor $R_{\mathrm{CT}}$ observed with the GCE-PAN-Ab-BSA immunosensor electrode is not surprising. Second, transition metallophthalocyanines $(\mathrm{MPc}=$ where $\mathrm{M}=\mathrm{Fe}, \mathrm{Co}, \mathrm{Cu}, \mathrm{Ni}$, etc. $)$ are known for their electrocatalytic/redox activities due to their central metal ions [24-29]; thus, the redox activity of the FePc in this work is not surprising. Third, the interaction of FePc with BSA in this work is highly possible for the following reasons: (i) BSA interacts and binds with a large variety of mononuclear and polynuclear metal ion complexes with aromatic ligands 
(such as transition MPc complexes such as FePc), a process which is made possible because BSA molecule consists of 583 amino acids, bound in a single chain cross-linked with 17 cystine residues $[30,31]$; (ii) BSA has a binding/adsorption affinity with Fe-based materials; for example, it interacts with steel and such adsorption has been reported to be accompanied by a fast charge-transfer [31]; (iii) BSA interacts with magnetic iron oxide nanoparticles, and such interaction is very spontaneous, i.e., negative change in the Gibb's free energy $(-\Delta G)[32])$; and (iv) the binding energy of BSA with transition metal ions is slightly smaller/less stable with some transition metal ions (such as $\mathrm{Fe}^{2+}, \mathrm{Ni}^{2+}, \mathrm{Co}^{2+}$ ) than others (such as $\mathrm{Cu}^{2+}$ and $\mathrm{Zn}^{2+}$ ) [33]. Fourth, the co-operative catalysis of BSA-FePc in this work is possible because (i) BSA has been reported to be a good catalyst only when it is conjugated with a co-catalyst such as an MPc complex (e.g., BSA-conjugated CuPc complex catalysed Diels-Alder reaction [34]), or iodine (e.g., co-operative cascade catalysis by BSA-iodine complex [35]). Thus, it can be concluded that the faster electron-transfer kinetics (electrocatalysis) observed for the GCE-PAN@FePc-Ab-BSA is related, not just to its high surface area and pore volume of the PAN@

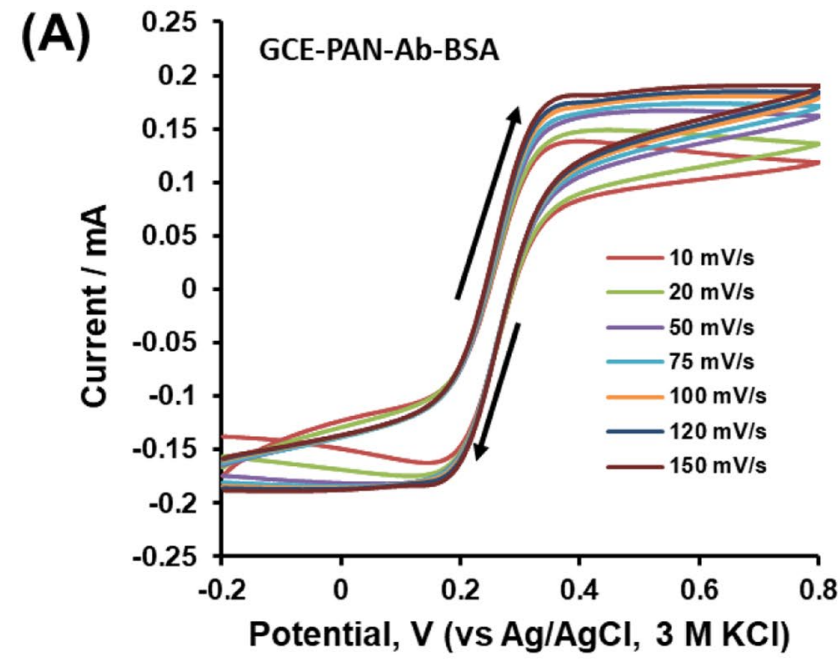

(C)
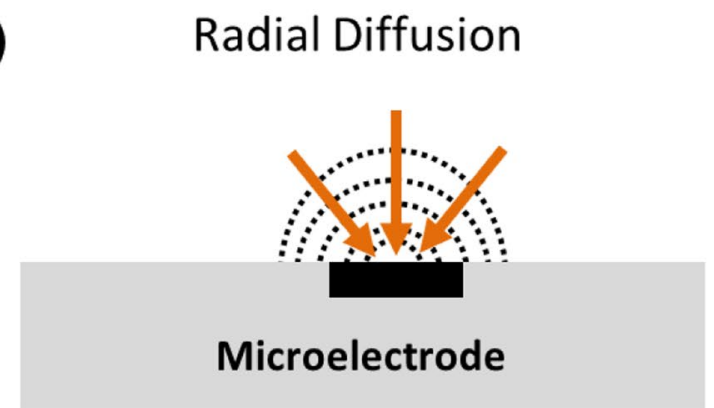

Fig. 5 Cyclic voltammetric responses of the electrochemical immunosensors conducted in redox probe solutions, $\left[\mathrm{Fe}(\mathrm{CN})_{6}\right]^{4-}$ / $\left[\mathrm{Fe}(\mathrm{CN})_{6}\right]^{3-}$, in PBS/AE (pH 7.4): (A) GCE-PAN-Ab-BSA and (B)
FePc compared to the PAN fibres, as confirmed by the BET data, but due to the co-operative interaction of FePc and BSA.

Next, we investigated the voltammetric behaviour of the two BSA-conjugated immunosensors (GCE-PAN-Ab-BSA and GCE-PAN@FePc-Ab-BSA) in the redox probe at various scan rates. As seen in Fig. 5, the GCE-PAN-Ab-BSA showed sigmoid-like voltammograms at every scan rate (Fig. 5A), which is characteristic of radial or convergent diffusion process in microelectrodes [36-38] (Fig. 5C). On the other hand, the GCE-PAN@FePc-Ab-BSA showed welldefined voltammetric response at every scan rate (Fig. 5B), characteristic of linear diffusion observed in macroelectrodes [21, 39, 40] (Fig. 5D).

Unlike the voltammograms observed in the PAN-based immunosensor, the peak current in PAN@FePc-based voltammograms showed direct proportionality with the square root of the scan rate, clearly confirming the planar diffusion process. The voltammetric responses at the PANbased immunosensor (micro-electrodes) are quite different from those observed at the PAN@FePc-based immunosensor (macro-electrodes) because at different scan rates, the
(B)

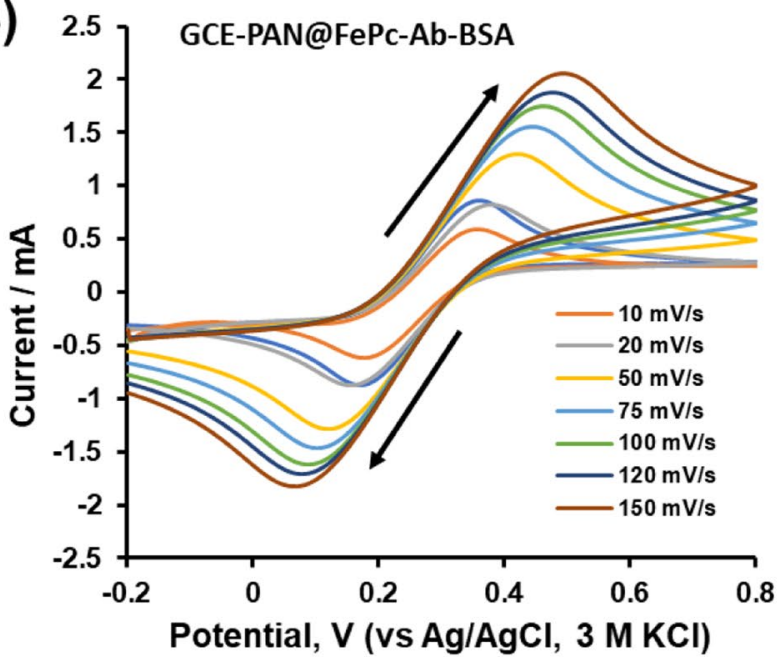

(D)

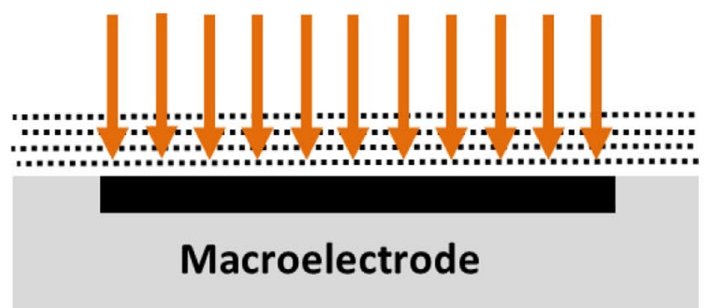

GCE-PAN@FePc-Ab-BSA at different scan rates, and their corresponding diffusion modes; (C) radial diffusion and (D) planar diffusion 
dimensions of the micro-electrode remain much smaller than the diffusion distance for molecules in solution. The dominance of planar diffusion in the macroelectrode's voltammetry and the dominance of radial diffusion in microelectrode's voltammetry are well described in the literature. The results again confirm the excellent mass transport and electron transfer kinetics of the GCE-PAN@FePc-Ab-BSA over its GCE-PAN-Ab-BSA counterpart.

\section{Immunosensing at GCE-PAN-Ab-BSA vs. GCE-PAN@ FePc-Ab-BSA: Vibrio cholerae Toxin as a Model/Case Study Bioanalyte}

Electrochemical immunosensing of Vibrio cholerae toxin (VCT) was conducted using EIS. Figure 6 shows typical Nyquist plots obtained at different concentrations of the VCT. The electroanalysis was conducted by casting $2 \mu \mathrm{L}$ of the VCT on the immunosensor surface and allowed to
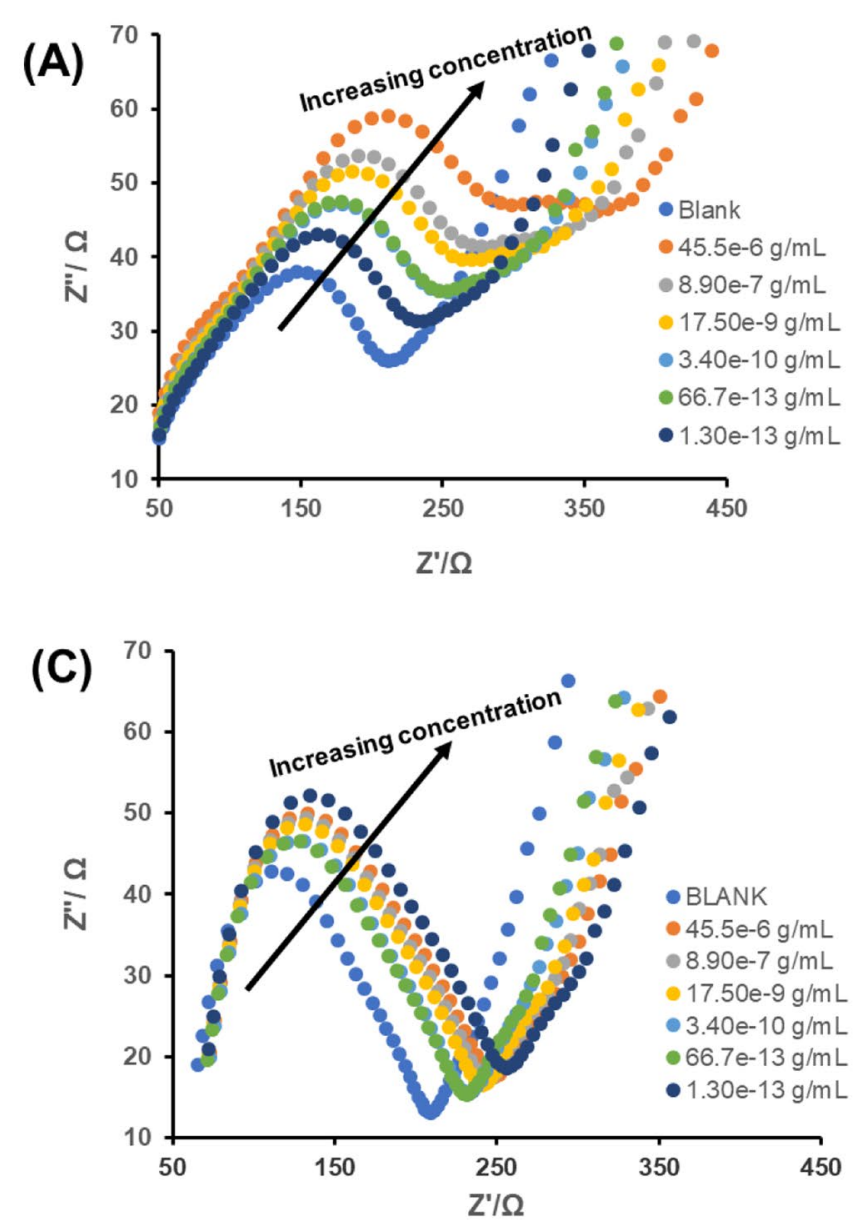

Fig. 6 EIS concentration study of the (A) GCE-PAN-Ab-BSA and (C) GCE-PAN@FePc-Ab-BSA, and ther respective calibration curves (B, D). The concentration range of VCT: $1.30 \times 10^{-13}$ to $45.5 \times 10^{-6} \mathrm{~g} /$ $\mathrm{ml}$. All EIS data were collected in the redox probe $\left(\left[\mathrm{Fe}(\mathrm{CN})_{6}\right]^{4-}\right.$ / incubate for 20 min (see Fig. 1). As summarized in Fig. 1, upon incubation of the VCT onto the immunosensor, the extent to which immobilized antibody interacts with the VCT is established by the inhibition or resistance to the one-electron redox kinetics of the probe $\left(\mathrm{Fe}(\mathrm{CN})_{6}\right]^{3+}$ / $\left[\mathrm{Fe}(\mathrm{CN})_{6}\right]^{4+}$ or simply the charge transfer resistance $\left(R_{\mathrm{CT}}\right)$. The linear calibration curves for the two electrochemical immunosensors are summarized in the equations below:

GCE-PAN-Ab-BSA $\quad R_{\mathrm{CT}} / \Omega=8.35 \pm 0.53 \log (\mathrm{VCT} / \mathrm{g}$ $\left.\mathrm{mL}^{-1}\right)+241.51 \pm 4.41\left(R^{2}=0.9839\right)$.

GCE-PAN@FePc-Ab-BSA $R_{\mathrm{CT}} / \Omega=4.16 \pm 0.24 \log (\mathrm{VCT} / \mathrm{g}$ $\left.\mathrm{mL}^{-1}\right)+249.44 \pm 1.97\left(R^{2}=0.9870\right)$.

Table 2 summarizes the analytical parameters of merit, including the linear concentration range (LCR), sensitivity, limit of detection (LoD), and limit of quantification (LoQ),
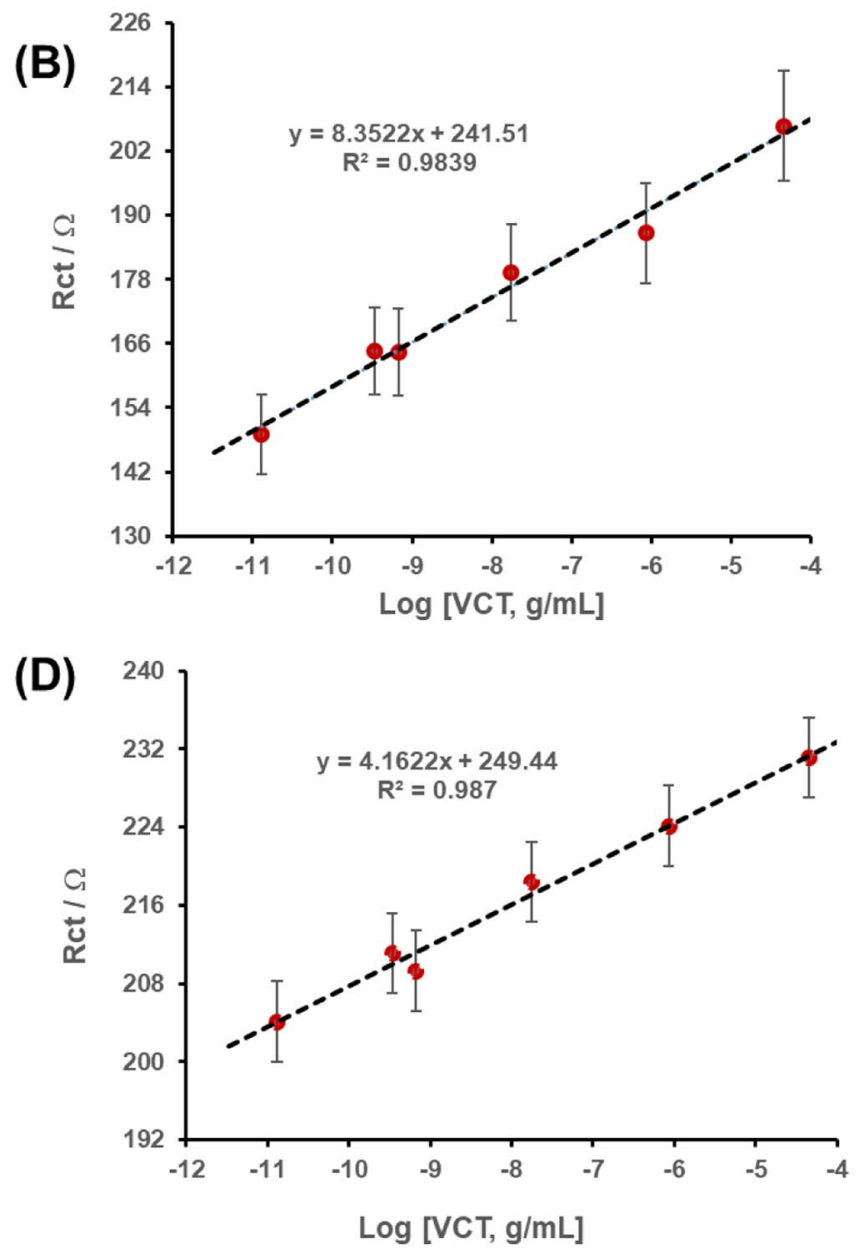

$\left[\mathrm{Fe}(\mathrm{CN})_{6}\right]^{3-}$ ) in PBS/AE ( $\left.\mathrm{pH} 7.4\right)$ at equilibrium potential of $0.13 \mathrm{~V}$. As the concentration increases, the resistance to electron-transfer $\left(R_{\mathrm{CT}}\right)$ increases (as depicted by the arrows) 
Table 2 Comparison of the electroanalytic parameters of merit for the detection of Vibrio cholerae as reported in the literature

\begin{tabular}{|c|c|c|c|c|}
\hline Immunosensor platform (technique) & $\mathrm{LCR}(\mathrm{g} / \mathrm{mL})$ & Sensitivity & $\mathrm{LoD}(\mathrm{g} / \mathrm{mL})$ & Ref \\
\hline GCE-PAN-Ab-BSA (EIS) & $1.3 \times 10^{-13}$ to $4.56 \times 10^{-5}$ & $16.12 \Omega / \log \left(\mathrm{VCT}, \mathrm{g} \mathrm{mL}^{-1}\right)$ & $3.2 \times 10^{-13}$ & This work \\
\hline GCE-PAN@FePc-Ab-BSA (EIS) & $1.3 \times 10^{-13}$ to $4.56 \times 10^{-5}$ & $4.16 \Omega / \log \left(\mathrm{VCT}, \mathrm{g} \mathrm{mL}^{-1}\right)$ & $2.0 \times 10^{-12}$ & This work \\
\hline LPEDOT-MWCNT (AdSWSV) & $10^{-14}$ to $10^{-7}$ & $7.21 \log [\mathrm{VCT}, \mathrm{g} / \mathrm{mL}]$ & $\sim 10^{-15}$ & 9 \\
\hline GCE-PPI-AuNP (SWV, EIS) & $10^{-7}$ to $10^{-12}$ & N/A & $7.2 \times 10^{-13}$ & 10 \\
\hline ZnO-ITO-Ab-BSA (DPV) & $12.5 \times 10^{-9}$ to $5.00 \times 10^{-7}$ & 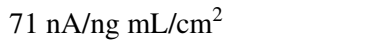 & $1.6 \times 10^{-10}$ & 11 \\
\hline ITO-PANnf-Ab-BSA (DPV) & $6.25 \times 10^{-9}$ to $5.00 \times 10^{-7}$ & $90 \mathrm{nA} / \mathrm{ng} \mathrm{mL} / \mathrm{cm}^{2}$ & $2.2 \times 10^{-10}$ & 12 \\
\hline GCE-Cu ${ }^{2+}$-pp-NTA-MWCNTs (EIS) & $10^{-13}$ to $10^{-5}$ & $24.7 \Omega$ per order of magnitude & $\sim 10^{-13}$ & 13 \\
\hline GCE-CNF-BSA (SWV, EIS) & $1.3 \times 10^{-13}$ to $4.56 \times 10^{-5}$ & $9.775 \Omega$ per order of magnitude & $1.25 \times 10^{-13}$ & 14 \\
\hline GCE-OLC@PAN-Ab-BSA (SWV (EIS) & $1.3 \times 10^{-13}$ to $4.56 \times 10^{-5}$ & $26.74 \log (\mathrm{VCT}, \mathrm{g} / \mathrm{mL})$ & $2.5 \times 10^{-17}$ & 15 \\
\hline
\end{tabular}

$C N F$ carbon nanofibers, $P P I-A u N P$ poly (propylene imine) dendrimer (PPI) and gold nanoparticles (AuNP), ZnO zinc oxide, ITO indium tin oxide, $P A N n f$ polyacrylonitrile nanofiber, $C u^{2+}-p p-N T A / M W C N T s$ Copper (II) complex with polypyrrole-nitrilotriacetic acid on multi-wall carbon nanotubes, GLI ganglioside-liposome immunoassay, FILIA flow injection liposome immunoanalysis, LPEDOT-MWCNT liposomes and poly(3,4-ethylenedioxythiophene)-coated carbon nanotubes, $A d S W S V$ adsorptive square-wave stripping voltammetry

for the two immunosensors in comparison with literature reports on similar immunosensors for the VCT. The LoD describes the concentrations of the VCT equal to 3 times the standard deviation of the y-intercept of the calibration curve above the blank signal under the same experimental conditions [14, 40]. Both immunosensors gave satisfactory LoD with the GCE-PAN-Ab-BSA giving the least LoD of $3.2 \times 10^{-13} \mathrm{~g} / \mathrm{mL}$ compared to that of the GCE-PAN@ FePc-Ab-BSA of $2.0 \times 10^{-12} \mathrm{~g} / \mathrm{mL}$. As compared in Table 2, only few reports gave lower LoD of $\sim 10^{-15} \mathrm{~g} / \mathrm{mL}[10]$ and $\sim 10^{-17} \mathrm{~g} / \mathrm{mL}$ [15], while the LoDs obtained in this work are comparable or even better than some reports [9, 11-14].

From the result, the insulating electrochemical immunosensor (i.e., GCE-PAN-Ab-BSA) provides the best immunosensing towards Vibrio cholerae compared to the conducting electrochemical immunosensor (i.e., GCE-PAN@FePc-Ab-BSA). The high performance of the PAN-based immunosensor is related to its redox-inactive nature (assisted by the efficient blocking of the non-specific sites by the BSA), while the PAN@FePc-based immunosensor is related to its redox-activity (assisted by the facile co-operative interaction of BSA with $\mathrm{FePc}$ ).

\section{Repeatability, Stability, and Specificity of the Electrochemical Immunosensors}

Considering the high performance of the GCE-PAN-AbBSA immunosensor, selectivity, repeatability, and stability studies were conducted with this immunosensor. The interferents (UA, CA, and AA) were used at high concentrations $(\sim 0.5 \mathrm{mg} / \mathrm{mL})$ compared to the VCT $(17.9 \mathrm{ng} / \mathrm{mL})$ (Fig. 7). Figure 7 shows that there is little or no difference in the SWV response in the presence and absence of the interfering
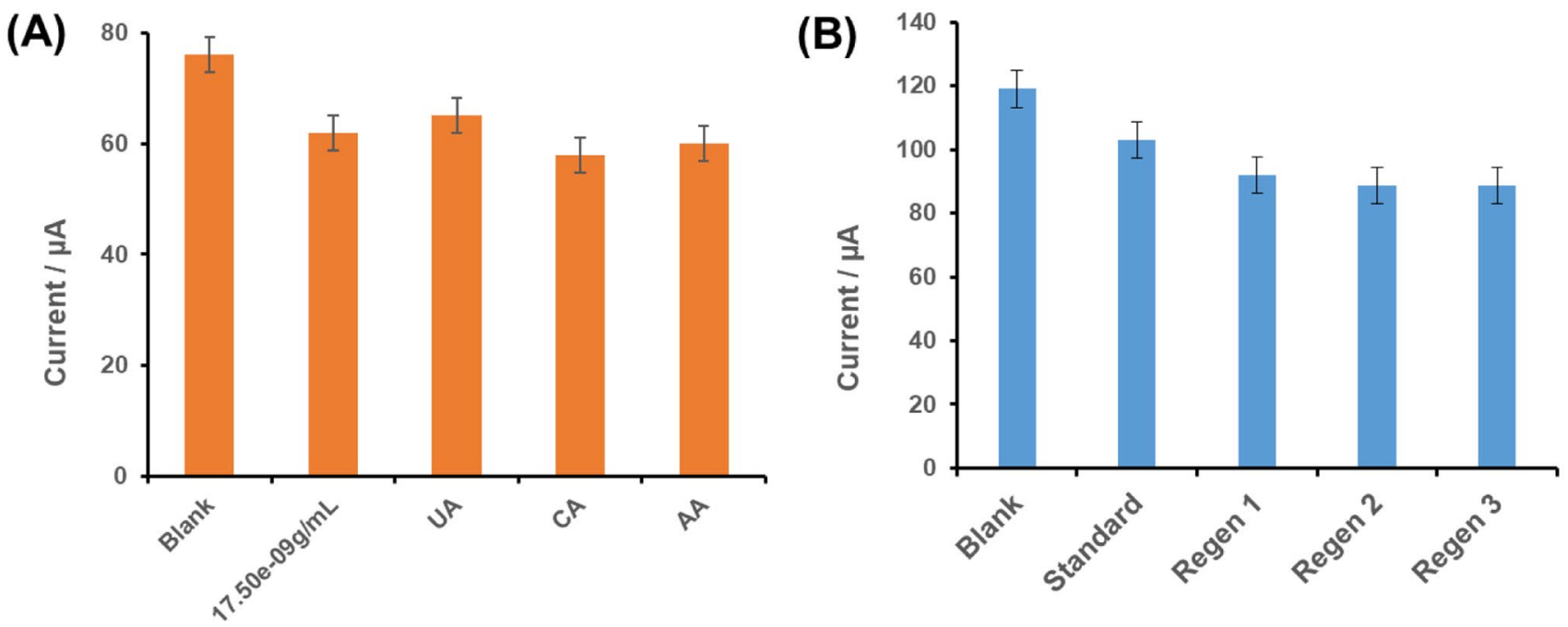

Fig. 7 (A) Selectivity and (B) re-usability studies of the GCE-PAN-Ab-BSA immunosensor. Note that "Regen" represents the regeneration of the immunosensor surface; the standard is VCT concentration $\left(4.85 \times 10^{-4} \mathrm{~g} / \mathrm{mL}\right)$ 
species. To determine the repeatability of use, the conventional method of analysis was adopted. First, the immunosensor was deployed to detect VCT (i.e., $8.90 \times 10^{-7} \mathrm{~g} /$ $\mathrm{mL}$ ). Then, the immunosensor was cleaned to wash off the VCT that was bound onto the surface by dipping it into a glycine $\mathrm{HCl}$ buffer solution ( $\mathrm{pH} 2.8$ ) for 5 min, and then deployed again to detect the VCT by repeating the processing of incubation and electrochemical testing in the redox probe solution. The whole process of immunosensor surfaceregeneration, re-incubation, and electrochemical testing was repeated five times to determine if there is any change in the electrochemical response from the initial immunosensor surface. The result shows about $13 \%$ loss in signal response from the original signal, meaning that the immunosensor shows satisfactory stability and can be used repeatedly without significant deterioration in signal response.

\section{Conclusions}

This work is an attempt to understand how BSA, a commonly used biomolecules in constructing immunosensors, can impact on the electrochemical detection when integrated with insulating PAN and conducting PAN@FePc electrode platforms, using Vibrio cholerae toxin as a model bioanalyte. Some unique findings should be emphasized. First, both PAN and PAN@FePc show different physico-chemical properties (in terms of morphology and BET surface areas and pore volumes). Second, the voltammetry of the BSA-conjugated PAN-based immunosensor was dominated by radial diffusion process (micro-electrode arrays), while that of the BSAconjugated PAN@FePc counterpart was dominated by planar diffusion process (macro-electrode). Third, both CV and EIS data prove that GCE-PAN-Ab-BSA is insulating (nonFaradaic), while the GCE-PAN@FePc-Ab-BSA is conducting/catalytic (aided by the facile co-ordination of BSA with $\mathrm{FePc}$ ). Fourth, the suppression of redox kinetics of the redox probe, which forms the underlying detection process for the antibody-antigen, was better observed with the GCE-PAN-AbBSA immunosensor than the conducting GCE-PAN@FePc$\mathrm{Ab}$-BSA immunosensor. The experimental findings prove that the insulating GCE-PAN-Ab-BSA immunosensor possesses the best characteristics for the detection of Vibrio cholerae toxin (in terms of sensitivity, LoD and LoQ). In general, one may conclude that the BSA-conjugation with the electrode platform can lead to different redox-chemistries, Faradaic or non-Faradaic. The immunosensitive detection is dependent on several factors including, but not limited to, surface area, pore volume, and nanoparticulate nature and redox activities.

Abbreviations Ag: Antigen; Ab: Anti-cholera toxin antibody; EDC: 1-Ethyl-3-(3-dimethylaminopropyl) carbodiimide hydrochloride; EDTA: Ethylenediaminetetraacetic acid; LoD: Limit of detection; PBS/
AE: Phosphate-buffered saline electrolyte containing sodium azide (as a preservative) and EDTA (for disentangling cells); PAN: Polyacrylonitrile fibre; PAN@FePc: Polyacrylonitrile fibre modified with iron (II) phthalocyanine; Sulfo-NHS: N-hydroxysulfosuccinimide (Sulfo-NHS); TCBS: Thiosulphate citrate bile salt

Acknowledgements OC Okoroike is grateful for the 2020 TUT Doctoral Scholarship Award and the Wits for permission to visit its School of Chemistry to conduct some of the experiments during the COVID19 lockdown.

Funding This work was supported by Tshwane University of Technology (TUT), University of the Witwatersrand (Wits), and the National Research Foundation.

\section{References}

1. A.L. Page, K.P. Alberti, V. Mondonge, J. Rauzier, M.L. Quilici, P.J. Guerin, Evaluation of a rapid test for the diagnosis of cholera in the absence of a gold standard. PLoS One 7, e37360 (2012)

2. C. Portet, G. Yushin, Y. Gogotsi, Electrochemical performance of carbon onions, nanodiamonds, carbon black and multiwalled nanotubes in electrical double layer capacitors. Carbon 45, 25112518 (2007)

3. M. Alam, N.A. Hasan, M. Sultana, G.B. Nair, A. Sadique, A.S.G. Faruque, H.P. Endtz, R.B. Sack, A. Huq, R.R. Colwell, H. Izumiya, M. Morita, H. Watanabe, A. Cravioto. J. Clin. Microbiol. 48, $3918(2010)$

4. D.A. Sack, R.B. Sack, G.B. Nair, A. Siddique, Cholera. Lancet 363, 223-233 (2004)

5. T. Ramamurthy, S.K. Bhattacharya, Y. Uesaka, K. Horigome, M. Paul, D. Sen, S.C. Pal, T. Takeda, Y. Takeda, G.B. Nair, Detection of cholera toxin gene in stool specimens by polymerase chain reaction: comparison with bead enzyme-linked immunosorbent assay and culture method for laboratory diagnosis of cholera. J. Clin Microbiol. 30, 1783 (1992)

6. T. Konry, Y. Heyman, S. Cosnier, K. Gorgy, R.S. Marks, Characterization of thin poly (pyrrole-benzophenone) film morphologies electropolymerized on indium tin oxide coated optic fibers for electrochemical and optical biosensing. Electrochim. Acta 53, 5128-5135 (2008)

7. W.H. Koch, W.L. Payne, B.A. Wentz, T.A. Cebula, Rapid polymerase chain reaction method for detection of Vibrio cholerae in foods. Appl. Environ. Microbiol. 59, 556-560 (1993)

8. S. Karapetis, G.P. Nikoleli, C.G. Siontorou, D. P. Nikolelis, N. Tzamtzis, N. Psaroudakis, Development of an electrochemical biosensor for the rapid detection of cholera toxin based on air stable lipid films with incorporated ganglioside GM1 using graphene electrodes. Electroanalysis 28, 1584 - 1590 (2016)

9. S. Viswanathan, L.C Wu, M.R. Huang, J.A.A. Ho, Electrochemical immunosensor for cholera toxin using liposomes and poly $(3,4-$ ethylenedioxythiophene)-coated carbon nanotubes. Anal. Chem. 78, 1115-1121 (2006).

10. P. Tshikalaha, O.A. Arotiba, Dendrimer Supported Electrochemical Immunosensor for the Detection of Cholera Toxin in Water. Int. J. Electrochem. Sci. 10, 10083-10092 (2015)

11. P.K. Gupta, Z.H. Khan, P.R. Solanki, One-step electrodeposited porous $\mathrm{ZnO}$ thin film based immunosensor for detection of Vibrio cholerae Toxin. J. Electrochem. Soc. 163, B309-B318 (2016)

12. P.K. Gupta, A. Gupta, S.R. Dhakate, Z.H. Khan, P.R. Solanki, Functionalized polyacrylonitrile-nanofiber based immunosensor 
or Vibrio cholerae detection. J. Appl. Polym. Sci. 44170 (2016). https://doi.org/10.1002/APP.44170

13. Q. Palomara, C. Gondrana, M. Holzingera, R. Marks, S. Cosnier, Controlled carbon nanotube layers for impedimetric immunosensors: high performance label free detection and quantification of anti-cholera toxin antibody Biosens. Bioelectron. 97, 177-183 (2017)

14. O.C. Ozoemena, T. Maphumulo, J.L. Shai, K.I. Ozoemena, Electrospun carbon nanofibers as an electrochemical immunosensing platform for vibrio cholerae toxin: aging effect of the redox probe ACS. Omega 5, 5762-5771 (2020)

15. O.C. Ozoemena, N.S. Mathebula, T.J. Ehirim, T. Maphumulo,G.M. Valipke, J. L. Shai, K.I. Ozoemena Onion-like carbon re-inforced electrospun polyacrylonitrile fibres for ultrasensitive electrochemical immunosensing of Vibrio cholerae Toxin. Electrochim. Acta 356, 136816 (2020)

16. H. Xiao, S. Wei, Z. Chen, L. Cao, Label-free electrochemical immunosensor based on gold and iron-oxide nanoparticle comodified rGO-TEPA hybrid for sensitive detection of carcinoembryonic antigen. Electrocatalysis 11, 513-521 (2021)

17. V. Pagliarini, D. Neagu, V. Scognamiglio, S. Pascale, G. Scordo, G. Volpe, E. Delibato, E. Pucci, A. Notargiacomo, M. Pea, D. Moscone, F. Arduini, Treated gold screen-printed electrode as disposable platform for label-free immunosensing of Salmonella Typhimurium. Electrocatalysis 10, 288-294 (2019)

18. N.S. Mathebula, J. Pillay, G. Toschi, J.A. Verschoor, K.I. Ozoemena, Recognition of anti-mycolic acid antibody at self-assembled mycolic acid antigens on a gold electrode: a potential impedimetric immunosensing platform for active tuberculosis. Chem. Commun. 3345-3347 (2009)

19. J. Xue, J. Xie, W. Liu, Y. Xia, Electrospun nanofibers: new concepts, materials, and applications. Acc. Chem. Res. 50, 19761987 (2017)

20. O.C. Ozoemena, L.J. Shai, T. Maphumulo, K.I. Ozoemena, Electrochemical sensing of dopamine using onion-like carbons and their carbon nanofiber composites. Electrocatalysis 10, 381-391 (2019)

21. L. Gaolatlhe, R. Barik. S.C.Ray, K.I.Ozoemena, Voltammetric responses of porous $\mathrm{Co} 3 \mathrm{O} 4$ spinels supported on MOF-derived carbons: effects of porous volume on dopamine diffusion processes. J. Electroanal. Chem. 2020, 113863

22. K.I. Ozoemena, N.S. Mathebula, J. Pillay, G. Toschi, J.A. Verschoor, Electron transfer dynamics across self-assembled $\mathrm{N}$-(2-mercaptoethyl) octadecanamide/mycolic acid layers: impedimetric insights into the structural integrity and interaction with anti-mycolic acid antibodies. Phys. Chem. Chem. Phys. 12, 345357 (2010)

23. M. Aydın, E.B. Aydın, M.K. Sezgintürk, A highly selective electrochemical immunosensor based on conductive carbon black and star PGMA polymer composite material for IL-8 biomarker detection in human serum and saliva Biosens. Bioelectronics 117, 720-728 (2018)

24. KI Ozoemena, T Nyokong, Synthesis, spectroscopy and photochemistry of octasubstituted thiol-derivatized phthalocyaninatozinc(II) complexes. Inorg. Chem. Commun. 6, 1192-1195 (2003)

25. K.I. Ozoemena, T. Nyokong, P. Westbroek, Self-assembled monolayers of cobalt and iron phthalocyanine complexes on gold electrodes: comparative surface electrochemistry and interaction with thiols and thiocyanate. Electroanalysis 15, 1762-1770 (2003)

26. K.I. Ozoemena, T. Nyokong, Photosensitized transformation of 4-chlorophenol in the presence of aggregated and non-aggregated metallophthalocyanines. Talanta 67, 162-168 (2005)

27. K.I. Ozoemena, T Nyokong, Comparative electrochemical and electrocatalytic studies of cobalt, iron and manganese phthalocyanine complexes axially coordinated to mercaptopyridine selfassembled monolayer at gold electrodes. Electrochim Acta 51, 2669-2677 (2006)

28. K.I. Ozoemena, T. Nyokong, D. Nkosi, I. Chambrier, M.J. Cook, Heterogeneous electron transfer kinetics and electrocatalytic behaviour of mixed self-assembled ferrocenes and SWCNT layers. Electrochim Acta 52, 4132-4143 (2007)

29. S.A. Mamuru, KI Ozoemena, Heterogeneous electron transfer and oxygen reduction reaction at nanostructured Iron(II) phthalocyanine and its MWCNTs nanocomposites. Electroanalysis 22, 985-994 (2010)

30. T. Topală, A. Bodoki, L. Oprean, R. Oprean, Bovine serum albumin interactions with metal complexes. Clujul Medical 87, 215-219 (2014)

31. S. Omanovic, S.G. Roscoe, Electrochemical studies of the adsorption behavior of bovine serum albumin on stainless steel. Langmuir 15, 8315-8321 (1999)

32. Q. Yang, J. Liang, H. Han, Probing the interaction of magnetic iron oxide nanoparticles with bovine serum albumin by spectroscopic techniques. J. Phys. Chem. B. 113, 10454-10458 (2009)

33. V.E. Atalay, I.B. Ölüç, M. Karahan, Modeling of BSA-metal ionacrylic acid complex by theoretical methods: semi-empirical PM6 and docking study. Acta Phys. Pol. A. 134, 1201 - 1203 (2018)

34. D.C.M. Albanese, N. Gaggero, Albumin as a promiscuous biocatalyst in organic synthesis. RSC Adv. 5, 10588 (2015)

35. Saima, D. Equbal, A.G. Lavekara, A.K. Sinha, Cooperative catalysis by bovine serum albumin-iodine towards cascade oxidative coupling-C (sp2)-H sulfenylation of indoles/hydroxyaryls with thiophenols on water. Org. Biomol. Chem. 14, 6111 (2016)

36. A.R.A. Rahman, A. Guiseppi-Elie, Design considerations in the development and application of microdisc electrode arrays (MDEAs) for implantable biosensors. Biomed. Microdevices 11, 701-710 (2009)

37. W.M. Damien, Arrigan, Tutorial review. Voltammetric determination of trace metals and organics after accumulation at modified electrodes. Analyst 129, 1157-1165 (2004)

38. D.A.C. Brownson, D.K. Kampouris, C.E. Banks, Graphene electrochemistry: fundamental concepts through to prominent applications. Chem. Soc. Rev. 41, 6944-6976 (2012)

39. I. Streeter, G.G. Wildgoose, L. Shao, R.G. Compton, Determination of cysteine in plasma and urine and homocysteine in plasma by high-pressure liquid chromatography. Sens. Actuators B. 133, 462-466 (2008)

40. P. Teengam, W. Siangproh, A. Tuantranont, C.S. Henry, T. Vilaivan, O. Chailapakul, Electrochemical paper-based peptide nucleic acid biosensor for detecting human papillomavirus. Anal. Chim. Acta 952, 32-40 (2017)

Publisher's Note Springer Nature remains neutral with regard to jurisdictional claims in published maps and institutional affiliations. 